\title{
Avaliação da frutosamina como parâmetro de controle glicêmico na gestante diabética
}

\author{
Evaluation of fructosamine as a parameter of blood \\ glucose control in diabetic pregnant women
}

Alina Coutinho Rodrigues Feitosa', Flávio Silva Andrade²

\begin{abstract}
1 Maternidade Professor José Maria de Magalhães Netto, Departamento de Obstetrícia e Ginecologia, Salvador, BA. Hospital Santa Izabel da Santa Casa de Misericórdia da Bahia, Departamento de Clínica Médica, Salvador, BA. Escola Bahiana de Medicina e Saúde Pública, Salvador, BA, Brazil ${ }^{2}$ Maternidade Professor José Maria de Magalhães Netto, Departamento de Obstetrícia e Ginecologia, Salvador, BA. Ambulatório de Endocrinologia da Maternidade Professor José Maria de Magalhães Netto, Salvador, BA, Brazil
\end{abstract}

\section{RESUMO}

Objetivo: Avaliar parâmetros alternativos para monitorar a glicemia em portadoras de diabetes na gestação estudando a relação entre a frutosamina e a automonitoração em gestantes portadoras de diabetes. Materiais e métodos: A frutosamina sérica e os parâmetros da automonitoração nos 14 dias que antecederam a coleta da frutosamina foram avaliados em 47 gestantes portadoras de diabetes. Resultados: Setenta e uma determinações de frutosamina e 2.238 glicemias capilares (GCs) foram analisadas. A frutosamina correlacionou-se com o índice de excursões hiperglicêmicas (HBGI) e o desvio-padrão das glicemias $(r=0,28 ; p=0,021$ e $r=0,26$; $p=0,03$, respectivamente). A comparação entre as mães dos neonatos com peso adequado ou grandes ao nascer com as genitoras que tiveram neonatos pequenos para a idade gestacional (PIG) revelou que estas tiveram menor média glicêmica (105 vs. 114 e 119 mg/dL), maior índice de excursões hipoglicêmicas $(5,8$ vs. 1,3 e 0,7) e maior percentual de hipoglicemias (11 vs. 0 e $0 \%$ ) mesmo com frutosamina dentro dos valores de referência (242 vs. 218 e $213 \mu \mathrm{mol} / \mathrm{l}$ ). Conclusão: A frutosamina pode ser utilizada como parâmetro auxiliar à automonitoração para avaliação de hiperglicemias e variabilidade glicêmica, entretanto pode subestimar hipoglicemias em gestantes com fetos PIG. Arq Bras Endocrinol Metab. 2014;58(7):724-30

\section{Descritores}

Frutosamina; controle; diabetes gestacional; diabetes melito; automonitoração da glicemia

\section{ABSTRACT}

Correspondência para: Alina Coutinho Rodrigues Feitosa Coordenação de Ensino e Pesquisa Maternidade Professor José Maria de Magalhães Netto

Rua Marques de Maricá, $s / n^{\circ}$ 41500-300 - Salvador, BA, Brasil alinafeitosa@yahoo.com.br

Recebido em 11/Set/2014 Aceito em 30/Jul/2014

DOI: $10.1590 / 0004-2730000002990$
Objective: To evaluate the alternative parameters to monitor glycemia in pregnant women with diabetes studying the relationship between fructosamine testing and self monitoring of blood glucose in pregnant women with diabetes. Materials and methods: Serum fructosamine levels and the self monitoring of blood glucose over 14 days before the collection of fructosamine were evaluated in 47 diabetic pregnant women. Results: Seventy-one fructosamine levels and 2,238 glucose measurements (CGs) were analysed. Levels of fructosamine correlated with high blood glucose index (HBGI) and the standard deviation of glycemias $(r=0.28 ; p=0.021$ and $r=$ $0.26 ; p=0.03$, respectively). The comparison between the mothers of the newborns with appropriated or large birthweight and those who gave birth to small newborns for their gestational age (SGA) showed that the latter had a lower glycemic mean (105 vs. 114 and $119 \mathrm{mg} / \mathrm{dL}$ ), a higher low blood glucose index (5.8 vs. 1.3 and 0.7 ) and a higher percentage of hyperglycemias ( 11 vs. 0 and $0 \%$ ) even when the fructosamine falls within the reference values (242 vs. 218 and $213 \mu \mathrm{mol} / \mathrm{l})$. Conclusion: The levels of fructosamine can be used as further parameter to aid self monitoring of blood glucose to evaluate hyperglycemias and glycemic variability, however, this can underestimate hypoglycemias in pregnant women carrying small-for-gestational age fetuses. Arq Bras Endocrinol Metab. 2014;58(7):724-30

\section{Keywords}

Fructosamine; control; gestational diabetes; diabetes mellitus; self-monitoring blood glucose 


\section{INTRODUÇÃO}

A

frutosamina é uma cetoamina derivada de uma reação não enzimática irreversível de um açúcar (grupo carbonil da glicose) com uma proteína (grupo amino da proteína) $(1,2)$ e é o termo geral para descrever as proteínas glicadas totais (1).

A frutosamina é aumentada em portadores de diabetes melito $(\mathrm{DM})$ em decorrência da formação de aldiminas instáveis e reversíveis que são posteriormente rearranjadas a cetoaminas irreversíveis e estáveis na presença de hiperglicemia persistente (2). É proporcional à concentração da glicemia (3) e correlaciona-se com a glicemia de jejum (4-6) e com a hemoglobina glicada (HbAlc) (4-6). A frutosamina pode ser utilizada como parâmetro auxiliar para o controle glicêmico de portadores de DM em situações nas quais a aplicabilidade da HbAlc é limitada, como hemoglobinopatias, hemólise, hemoglobina carbamilada e anemia $(7,8)$. Por ser um produto de glicação não enzimática das proteínas séricas, em sua maioria a albumina $(50-80 \%)(1,2,9)$, a frutosamina representa a concentração média da glicose em três semanas (8) e é um parâmetro alternativo à automonitoração da glicemia capilar para estimar $\mathrm{o}$ controle glicêmico a curto prazo (2). Adicionalmente, correlaciona-se com a média pós-prandial máxima da glicose (MPMG), área sob a curva para glicemia superior a $180 \mathrm{mg} / \mathrm{dL}$ (AUC-180) e a média da amplitude das excursões glicêmicas (MAGE) (10) que representam, respectivamente, os dois primeiros, a hiperglicemia pós-prandial e o último, a variabilidade glicêmica. Em grupos especiais, como portadores de nefropatias em programa de diálise, a frutosamina é útil para avaliar o controle glicêmico e se relaciona com desfechos clínicos e mortalidade (11). Em portadoras de diabetes na gestação, nas quais a necessidade de controle glicêmico rigoroso é imperativo para prevenção de complicações materno-fetais (12) e ajustes frequentes nas doses de insulinas são indispensáveis, a frutosamina tem sido utilizada para avaliar o controle glicêmico a curto prazo. Entretanto, os estudos que avaliam a frutosamina na gestação como parâmetro de controle glicêmico têm resultados controversos (13-17).

Identificar a utilidade da frutosamina como parâmetro auxiliar no controle glicêmico de portadoras de diabetes na gestação pode ser útil especialmente naquelas em que a automonitoração da glicemia capilar não está disponível ou limitada. O presente estudo avalia a associação entre as concentrações de frutosamina e variáveis da automonitoração da glicemia capilar em portadoras de diabetes durante a gestação e procura definir a importância da frutosamina como parâmetro de controle glicêmico na gestação.

\section{MATERIAIS E MÉTODOS}

\section{Desenho do estudo}

Realizou-se um estudo transversal analítico, com coleta retrospectiva de dados. Os sujeitos da pesquisa foram mulheres portadoras de diabetes na gestação que fizeram pré-natal no ambulatório de endocrinopatias na gestação da maternidade pública Professor José Maria de Magalhães Neto, em Salvador, Estado da Bahia, referência em gestação de alto risco, no período de setembro de 2010 a junho de 2012. O protocolo do estudo foi aprovado pelo Comitê de Ética.

Foram incluídas gestantes entre 18 e 45 anos portadoras de diabetes pré-gestacional e gestacional que tinham dados de glicemias capilares da automonitoração nos 14 dias que antecederam a coleta da frutosamina e que foram acompanhadas até o parto. Excluíram-se as gestantes que tinham dados de automonitoração incompletos. O diabetes pré-gestacional foi representado pelas portadoras de diabetes melito do tipo 1 e 2 e o diabetes gestacional, definido conforme os critérios da Associação Americana de Diabetes (18). Todas as gestantes foram acompanhadas por obstetras, endocrinologistas, enfermeiras e nutricionistas durante o pré-natal. As consultas com nutricionistas eram mensais, com endocrinologistas eram quinzenais e com os demais profissionais, quinzenais a mensais.

A coleta dos dados foi feita por meio de revisões do prontuário eletrônico do ambulatório e do registro eletrônico das glicemias capilares em programa de gerenciamento de diabetes. Os dados antropométricos, demográficos, clínicos e laboratoriais foram extraídos dos registros em prontuário eletrônico. $\mathrm{O}$ peso do recém-nato $(\mathrm{RN})$ ao nascimento foi classificado de acordo com a escala de Lubchenco (19), que considera como pequenos para a idade gestacional (PIG) os RNs com peso abaixo do percentil 10 e grandes para a idade gestacional (GIG) os RNs com peso acima do percentil 90. Foram denominados pré-termo os RNs nascidos com menos de 37 semanas.

A frutosamina sérica foi mensurada por ensaio colorimétrico com reagente azul de nitrotetrazol utilizando o kit comercial da BioTécnica com valores de referência de 205 a $285 \mu \mathrm{mol} / \mathrm{L}$ e coeficiente de variação de 
3,94\%. A HbAlc foi mensurada pela cromotografia líquida de alta perfomance com o valor de referência de 4 a $6 \%$.

As gestantes realizaram a automonitoração das glicemias logo antes e duas horas após o café da manhã, almoço, jantar e às 22 horas e às 3 horas. A faixa terapêutica-alvo do controle glicêmico foi de 60 a $126 \mathrm{mg} / \mathrm{dL}$ $(20,21)$ e hipoglicemia foi definida como valor inferior a $60 \mathrm{mg} / \mathrm{dL}$, adaptado de Crowther e cols. (20). As glicemias capilares da automonitoração mensuradas nas duas semanas que antecederam a coleta da frutosamina e armazenadas nos glicosímetros eram transmitidas, por bluetooth, para um programa de gerenciamento do diabetes a cada consulta endocrinológica. Esse programa calcula a média glicêmica, o percentil de glicemias acima, dentro e fora da faixa-alvo e os parâmetros da variabilidade glicêmica como o desvio-padrão (DP) das glicemias, o low blood glucose index (LBGI) e o high blood glucose index (HBGI).

O LBGI e o HBGI indicam, respectivamente, o risco de excursões hipoglicêmicas e hiperglicêmicas. As fórmulas para o cálculo já foram descritas (22-25) e validadas para uso clínico. Em resumo, o LBGI e o HBGI são números não negativos cuja soma varia de 0 a 100. Os índices são baseados na transformação não linear da escala de glicemia, aplicando-se simetria para a distribuição das leituras de glicemias de um indivíduo. As categorias de risco extraídas a partir desses índices são: LBGI, mínimo (LBGI $\leq 1,1)$, baixo $(1,1<$ LBGI $\leq 2,5)$, moderado $(2,5<\mathrm{LBGI} \leq 5)$, e alto (LBGI $>$ $5,0)(26)$; HBGI, baixo (HBGI $\leq 4,5)$, moderado $(4,5$ $<\mathrm{HBGI} \leq 9,0)$, e alto $(\mathrm{HBGI}>9,0)(24)$.

\section{Análise estatística}

As análises foram realizadas pelo Software SPSS (Statistical Package for the Social Sciences), versão 20.0. Os dados foram apresentados como médias \pm desvios-padrão (DP), mediana e variação, de acordo com a distribuição de normalidade. Variáveis qualitativas foram apresentadas como valor absoluto e percentual. Coeficientes de correlação de Pearson foram calculados para avaliar a relação entre as variáveis contínuas quando a distribuição fosse normal. Utilizou-se o coeficiente de correlação de Spearman quando a distribuição foi não paramétrica. O teste $t$ de Student foi utilizado para comparação das médias e o teste U de Mann Whitney, para comparação de variáveis contínuas, independentes e de distribuição não paramétrica. $O$ valor de $\mathrm{p}<0,05$ foi considerado estatisticamente significativo.
Os dados faltantes para as variáveis foram: tipo de diabetes $(19,6 \%)$, medicação usada para tratamento do diabetes $(13,9 \%)$ e tipo de parto $(21,7 \%)$. Dentre as 47 gestantes acompanhadas, 11 neonatos não tinham $\mathrm{o}$ peso registrado ao nascimento (parto fora da maternidade ou dado de peso faltante no registro).

\section{RESULTADOS}

Foram avaliadas 71 determinações de frutosamina e 2.238 glicemias capilares extraídas da automonitoração de 47 gestantes nas duas semanas que antecederam a coleta da frutosamina.

A tabela 1 mostra as características demográficas, clínicas e de tratamento das gestantes e informações sobre o parto e o RN.

Tabela 1. Características demográficas, clínicas e de tratamento das gestantes e informações sobre o parto e o recém-nato $(n=47)$

\begin{tabular}{|c|c|c|c|}
\hline & Média — DP & $\begin{array}{c}\text { Mediana } \\
\text { (variação) }\end{array}$ & n (\%) \\
\hline Idade (anos) & $32,2 \pm 6,2$ & $32,6(19-44,9)$ & \\
\hline Tipo DM* & & & $\begin{array}{c}3(7,9) \text { tipo } 1 \\
20(52,6) \text { tipo } 2 \\
15(39,5) \text { DMG }\end{array}$ \\
\hline $\begin{array}{l}\text { IG (na data da } \\
\text { coleta da } \\
\text { frutosamina) }\end{array}$ & $29 \pm 6,4$ & $30(14-39)$ & \\
\hline $\begin{array}{l}\text { Frutosamina } \\
(\mu \mathrm{mol} / \mathrm{L})\end{array}$ & $218 \pm 33,2$ & $218(147-306)$ & \\
\hline Tratamento $\mathrm{DM}^{* *}$ & & & $\begin{array}{c}18(50) \text { insulina } \\
13(36,1) \\
\text { insulina + } \\
\text { metformina } \\
3(8,3) \text { dieta } \\
2(5,6) \\
\text { metformina }\end{array}$ \\
\hline $\begin{array}{l}\text { Peso RN }{ }^{\dagger} \\
\text { (gramas) }\end{array}$ & $3170,8 \pm 743$ & $\begin{array}{c}3290 \\
(890-4584)\end{array}$ & \\
\hline $\begin{array}{l}\text { IG parto }^{\dagger} \\
\text { (semanas) }\end{array}$ & $37,6 \pm 2,0$ & $38(31-40)$ & \\
\hline Tipo parto $^{\dagger}$ & & & $\begin{array}{c}27 \text { (75) Cesário } \\
9 \text { (25) Natural }\end{array}$ \\
\hline $\begin{array}{l}\text { Prematuros }{ }^{\dagger}(\mathrm{GG} \\
\leq 37 \mathrm{~s})\end{array}$ & & & $10(27,8)$ \\
\hline \multicolumn{4}{|l|}{ Neonatos ${ }^{\dagger}$} \\
\hline$P I G$ & & & $4(11,1)$ \\
\hline AlG & & & $24(66,7)$ \\
\hline $\mathrm{GIG}$ & & & $08(22,2)$ \\
\hline
\end{tabular}

${ }^{*} n=38 ;{ }^{*} n=36 ; \uparrow n=36$; DP: desvio-padrão; DM: diabetes melito; DMG: diabetes melito gestacional; IG: idade gestacional; RN: recém-nato; PIG: pequeno para a idade gestacional; AIG: adequado para a idade gestacional; GIG: grande para a idade gestacional. 
Na tabela 2 estão os dados sobre o controle glicêmico, a frutosamina e as informações sobre o parto de acordo com o tipo de diabetes. A frutosamina não foi diferente entre as portadoras de diabetes do tipo 1 e 2 e DMG $(\mathrm{p}=0,06)$.

Não encontramos correlação entre a frutosamina e a média glicêmica, peso do RN e com as médias das glicemias pré e pós-prandiais.

A frutosamina correlacionou-se com o DP das glicemias $(\mathrm{r}=0,28 ; \mathrm{p}=0,02 \mathrm{l})$, com o HBGI $(\mathrm{r}=0,26 ; \mathrm{p}=$ $0,03)$ e com a HbAlc $(r=0,44 ; \mathrm{p}<0,0001)$.

$\mathrm{O}$ peso do $\mathrm{RN}$ correlacionou-se com a média glicêmica $(r=0,33 ; p=0,015)$, o LBGI $(r=-0,40$; $\mathrm{p}=0,003)$, o percentual de glicemias abaixo de 60 $\mathrm{mg} / \mathrm{dL}(\mathrm{r}=-0,50 ; \mathrm{p}<0,0001)$ e a glicemia média em jejum $(\mathrm{r}=0,33 ; \mathrm{p}=0,025)$ e pós-jantar $(\mathrm{r}=0,34 ; \mathrm{p}$ $=0,02)$.
As mães dos RNs nascidos pequenos para a idade gestacional tiveram menor média glicêmica, maior LBGI e mais hipoglicemias que os neonatos nascidos adequados para a idade gestacional e os grandes para a idade gestacional, ainda que os três grupos tivessem valores semelhantes de frutosamina. As associações entre as variáveis da automonitoração e a frutosamina em relação ao peso fetal estão na tabela 3 .

\section{DISCUSSÃO}

No presente estudo, a frutosamina, avaliada após duas semanas de automonitoração glicêmica em gestantes portadoras de diabetes, não se relacionou com a média glicêmica e as médias das glicemias pré ou pós-prandiais. Correlação significativa foi encontrada com o desvio-padrão das glicemias e o HBGI.

Tabela 2. Controle glicêmico, frutosamina, idade e peso do recém-nato de acordo com o tipo de diabetes (expressos em média \pm desvio-padrão e em mediana e variação)

\begin{tabular}{|c|c|c|c|c|c|}
\hline & Todas $(n=71)$ & DM1 $(n=6)$ & DM2 (n = 35) & DMG $(n=19)$ & $N C(n=11)$ \\
\hline Idade (anos) & $\begin{array}{c}32 \pm 6 \\
33(19-45)\end{array}$ & $\begin{array}{c}24 \pm 6 \\
20(19-32)\end{array}$ & $\begin{array}{c}34 \pm 4 \\
33(24-42)\end{array}$ & $\begin{array}{c}33 \pm 7 \\
33(23-43)\end{array}$ & $\begin{array}{c}33 \pm 8 \\
31(23-45)\end{array}$ \\
\hline Frutosamina ( $\mu \mathrm{mol} / \mathrm{L})$ & $\begin{array}{c}221 \pm 34 \\
218(147-327)\end{array}$ & $\begin{array}{c}242 \pm 15 \\
248(221-257)\end{array}$ & $\begin{array}{c}224 \pm 35 \\
218(171-306)\end{array}$ & $\begin{array}{c}210 \pm 28 \\
208(147-254)\end{array}$ & $\begin{array}{c}219 \pm 42 \\
213(174-327)\end{array}$ \\
\hline IG (sem) frutosamina & $\begin{array}{l}28,4 \pm 6,7 \\
30(8-39)\end{array}$ & $\begin{array}{c}29,7 \pm 7,9 \\
29(20-39)\end{array}$ & $\begin{array}{l}26,7 \pm 7,3 \\
28(8-38)\end{array}$ & $\begin{array}{c}30 \pm 4,7 \\
32(19-36)\end{array}$ & $\begin{array}{c}31,1 \pm 5,8 \\
30(21-39)\end{array}$ \\
\hline n glicemias* & $\begin{array}{c}32 \pm 20 \\
31(1-103)\end{array}$ & $\begin{array}{c}29 \pm 8 \\
30(20-39)\end{array}$ & $\begin{array}{c}38 \pm 24 \\
38(1-103)\end{array}$ & $\begin{array}{c}24 \pm 14 \\
21(2-54)\end{array}$ & $\begin{array}{c}26 \pm 15 \\
27(9-45)\end{array}$ \\
\hline $\begin{array}{l}\text { Média glicêmica } \\
(\mathrm{mg} / \mathrm{dL})\end{array}$ & $\begin{array}{c}115 \pm 19 \\
114(81-167)\end{array}$ & $\begin{array}{c}115 \pm 25 \\
124(82-144)\end{array}$ & $\begin{array}{c}110 \pm 15 \\
109(88-154)\end{array}$ & $\begin{array}{c}117 \pm 23 \\
116(81-167)\end{array}$ & $\begin{array}{c}125 \pm 18 \\
122(103-166)\end{array}$ \\
\hline DP das glicemias & $\begin{array}{c}34 \pm 16 \\
31(9-92)\end{array}$ & $\begin{array}{c}55 \pm 29 \\
45(26-92)\end{array}$ & $\begin{array}{c}32 \pm 9 \\
31(9-52)\end{array}$ & $\begin{array}{c}29 \pm 13 \\
27(12-62)\end{array}$ & $\begin{array}{c}40 \pm 19 \\
32(19-83)\end{array}$ \\
\hline LBGI & $\begin{array}{c}2,6 \pm 2,7 \\
1,6(1-11)\end{array}$ & $\begin{array}{c}6,1 \pm 4,3 \\
6,2(0,4-11)\end{array}$ & $\begin{array}{c}2,7 \pm 2,5 \\
2,1(0-10,5)\end{array}$ & $\begin{array}{l}1,5 \pm 1,7 \\
0,7(0-6)\end{array}$ & $\begin{array}{c}2,3 \pm 2,8 \\
0,8(0,1-7,1)\end{array}$ \\
\hline $\mathrm{HBGl}$ & $\begin{array}{c}2,6 \pm 2,7 \\
1,6(0-11)\end{array}$ & $\begin{array}{c}3,3 \pm 3,2 \\
2,6(0,2-7,3)\end{array}$ & $\begin{array}{l}1,2 \pm 1,1 \\
0,9(0-6)\end{array}$ & $\begin{array}{c}1,6 \pm 2 \\
1(0-7,9)\end{array}$ & $\begin{array}{c}2,7 \pm 2,8 \\
1,8(0,3-8,3)\end{array}$ \\
\hline Acima do alvo** (\%) & $\begin{array}{c}31 \pm 20 \\
29(0-100)\end{array}$ & $\begin{array}{c}33 \pm 17 \\
39(8-51)\end{array}$ & $\begin{array}{c}28 \pm 19 \\
25(0-100)\end{array}$ & $\begin{array}{c}34 \pm 24 \\
38(0-75)\end{array}$ & $\begin{array}{c}31 \pm 20 \\
29(0-100)\end{array}$ \\
\hline Dentro do alvo** (\%) & $\begin{array}{c}62 \pm 20 \\
63(0-100)\end{array}$ & $\begin{array}{c}51 \pm 14 \\
56(33-66)\end{array}$ & $\begin{array}{c}65 \pm 20 \\
68(0-100)\end{array}$ & $\begin{array}{c}65 \pm 22 \\
63(25-100)\end{array}$ & $\begin{array}{c}54 \pm 17 \\
55(22-89)\end{array}$ \\
\hline Hipo $<60$ mg/dL & $\begin{array}{l}4,3 \pm 7,5 \\
0(0-29)\end{array}$ & $\begin{array}{l}15,1 \pm 11 \\
18(0-29)\end{array}$ & $\begin{array}{l}3,9 \pm 6,8 \\
0(0-28)\end{array}$ & $\begin{array}{c}1,3 \pm 4 \\
0(0-15)\end{array}$ & $\begin{array}{c}5 \pm 8 \\
0(0-22)\end{array}$ \\
\hline HbA1c (\%) & $\begin{array}{l}6,2 \pm 1,6 \\
6(3-14)\end{array}$ & $6,4 \dagger$ & $\begin{array}{c}6,4 \pm 1,9 \\
6,2(3-14)\end{array}$ & $\begin{array}{c}5,8 \pm 1,2 \\
6(3,3-8,2)\end{array}$ & $\begin{array}{c}6,2 \pm 1,2 \\
5,6(5,1-9,1)\end{array}$ \\
\hline Peso RN (g) & $\begin{array}{c}3263 \pm 696 \\
3500(890-4584)\end{array}$ & $\begin{array}{c}3283 \pm 670 \\
3500(2450-3900)\end{array}$ & $\begin{array}{c}3122 \pm 780 \\
3390(890-3990)\end{array}$ & $\begin{array}{c}3302 \pm 517 \\
3340(2280-4100)\end{array}$ & $\begin{array}{c}3585 \pm 676 \\
3650(2320-4584)\end{array}$ \\
\hline IG nasc (sem) & $\begin{array}{c}37,9 \pm 1,8 \\
38(31-40)\end{array}$ & $\begin{array}{c}38,3 \pm 0,2 \\
38,4(38-38,5)\end{array}$ & $\begin{array}{c}37,5 \pm 2,3 \\
38,2(31-39)\end{array}$ & $\begin{array}{c}37,9 \pm 1,3 \\
38(35-39)\end{array}$ & $\begin{array}{c}38,8 \pm 1,1 \\
39(37-40)\end{array}$ \\
\hline
\end{tabular}

* Número de glicemias capilares feitas 14 dias antes da determinação da frutosamina; ** alvo glicêmico: 60 a 126 mg/dL; ${ }^{\dagger}$ apenas uma gestante tinha a HbA1c mensurada; DM1: diabetes melito tipo 1; DM2: diabetes melito tipo 2; DMG: diabetes melito gestacional; NC: diabetes não classificado; IG: idade gestacional; DP: desvio-padrão; HBGI: high blood glucose index, LBGI: low blood glucose index, RN: recém-nato. 
Tabela 3. Comparação entre variáveis da automonitoração glicêmica e frutosamina entre os recém-natos classificados quanto ao peso do nascimento $(n=36)$

\begin{tabular}{|c|c|c|c|}
\hline & $\begin{array}{c}\text { PIG } \\
(n=4)\end{array}$ & $\begin{array}{c}\text { AIG } \\
(n=24)\end{array}$ & $\begin{array}{c}\text { GIG } \\
(n=8)\end{array}$ \\
\hline $\begin{array}{l}\text { Frutosamina } \\
(\mu \mathrm{mol} / \mathrm{l})\end{array}$ & $\begin{array}{c}242 \\
(174-275)\end{array}$ & $\begin{array}{c}218 \\
(166-327)\end{array}$ & $\begin{array}{c}213 \\
(147-257)\end{array}$ \\
\hline $\begin{array}{l}\text { Idade materna } \\
\text { (anos) }\end{array}$ & $\begin{array}{c}26^{\dagger} \\
(19-32)\end{array}$ & $\begin{array}{c}33 \\
(23-42)\end{array}$ & $\begin{array}{c}32 \\
(20-45)\end{array}$ \\
\hline Peso neonatal (g) & $\begin{array}{c}2285^{\dagger} \\
(890-2450)\end{array}$ & $\begin{array}{c}3222 \\
(1360-3700)\end{array}$ & $\begin{array}{c}3905^{\star} \\
(3500-4580)\end{array}$ \\
\hline IG (semanas) & $\begin{array}{c}37 \\
(31-38)\end{array}$ & $\begin{array}{c}38 \\
(31-40)\end{array}$ & $\begin{array}{c}39 \\
(38-40)\end{array}$ \\
\hline $\begin{array}{l}\text { Média glicêmica } \\
\text { (mg/dL) }\end{array}$ & $\begin{array}{c}105^{\dagger \star} \\
(82-107)\end{array}$ & $\begin{array}{c}114 \\
(87-154)\end{array}$ & $\begin{array}{c}119 \\
(81-166)\end{array}$ \\
\hline LBGI & $\begin{array}{c}5,8^{{ }^{\star}} \\
(2,8-10,1)\end{array}$ & $\begin{array}{c}1,3 \\
(0-7,1)\end{array}$ & $\begin{array}{c}0,7 \\
(0,1-6)\end{array}$ \\
\hline $\begin{array}{l}\text { Hipoglicemias < } 60 \\
\mathrm{mg} / \mathrm{dL}(\%)\end{array}$ & $\begin{array}{c}11^{1 *} \\
(3-23)\end{array}$ & $\begin{array}{c}0 \\
(0-22)\end{array}$ & $\begin{array}{c}0 \\
(0-15)\end{array}$ \\
\hline HBGl & $\begin{array}{c}0,6 \\
(0,2-1,2)\end{array}$ & $\begin{array}{c}0,8 \\
(0-8,3)\end{array}$ & $\begin{array}{c}1,3 \\
(0-8)\end{array}$ \\
\hline DP & $\begin{array}{c}32 \\
(31-39)\end{array}$ & $\begin{array}{c}30 \\
(13-83)\end{array}$ & $\begin{array}{c}29 \\
(19-59)\end{array}$ \\
\hline HbA1c (\%) & $\begin{array}{c}6,7 \\
(5,5-14,1)\end{array}$ & $\begin{array}{c}6 \\
(3,3-9,1)\end{array}$ & $\begin{array}{c}5,8 \\
(5,4-7,9)\end{array}$ \\
\hline $\begin{array}{l}\text { Glicemias capilares } \\
\text { (em } 15 \text { dias) }\end{array}$ & $\begin{array}{c}51^{\dagger} \\
(35-71)\end{array}$ & $\begin{array}{c}32 \\
(2-57)\end{array}$ & $\begin{array}{c}27 \\
(9-54)\end{array}$ \\
\hline
\end{tabular}

IG: idade gestacional; PIG: pequeno para a idade gestacional; AIG: adequado para a idade gestacional; GIG: grande para a idade gestacional; LGBI: low blood glucose index, HBGl: high blood glucose index; DP: desvio-padrão; $\uparrow p<0,05$ entre PIG e AIG; * $p<0,05$ entre GIG e AIG.
Apesar de existirem estudos que utilizaram a frutosamina como parâmetro para monitoração do controle glicêmico na gestante portadora de diabetes $(13,27,28)$, há divergentes informações sobre a associação da frutosamina com os parâmetros da automonitoração glicêmica, limitando sua utilização na prática. A maior parte desses estudos avaliou um número pequeno de gestantes portadoras de diabetes $(<50)$ e estudou apenas a correlação da frutosamina com a média glicêmica ou glicemia em jejum (vide tabela 4). No presente estudo, não encontramos relação entre a frutosamina e a média glicêmica. Esse achado está em concordância com estudos que avaliaram a relação frutosamina e parâmetros da SMBG e que não encontraram correlação com a média glicêmica (14) ou não acrescentaram vantagem adicional como índice de controle (29). Alguns estudos encontraram correlação fraca a moderada (15-17,30,31) com a média glicêmica, e a falta de forte correlação entre a média glicêmica nas últimas duas semanas e a frutosamina pode indicar que as concentrações de glicemia variam significativamente durante o dia e ao longo dos dias e a frutosamina não refletiria, adequadamente, os perfis glicêmicos diários. A divergência na força das correlações entre os estudos pode ser justificada por diferenças entre as amos-

Tabela 4. Estudos que avaliaram a frutosamina e parâmetros de controle glicêmico em gestantes portadoras de diabetes

\begin{tabular}{|c|c|c|c|c|}
\hline Autor & n e tipo de diabetes & $\begin{array}{c}\text { Parâmetro glicêmico } \\
\text { avaliado }\end{array}$ & Condição & $\begin{array}{c}\text { Correlação } \\
\text { frutosamina } r(p)\end{array}$ \\
\hline Pasi e cols. (1989) (30) & 41 DM (insulina) & Média glicêmica (plasma) & $\begin{array}{c}\text { Glicemia mensurada junto com } \\
\text { a frutosamina (corrigida por } \\
\text { albumina) }\end{array}$ & $0,52(p<0,01)$ \\
\hline Watson e cols. (1991) (31) & 50 DM (insulina) & Média pré-prandial & $\begin{array}{l}\text { SMBG por duas semanas } \\
\text { antes da frutosamina }\end{array}$ & $0,37(p<0,01)$ \\
\hline Koskinen e cols. (1992) (14) & 22 DMG e DMPG (insulina) & Média glicêmica & $\begin{array}{c}\text { SMBG por duas semanas } \\
\text { antes }\end{array}$ & 0,003 (NS) \\
\hline Parfitt e cols. (1993) (17) & 16 DM1 (insulina) & Média glicêmica & $\begin{array}{c}\text { SMBG por duas semanas } \\
\text { antes }\end{array}$ & $0,5(p<0,01)$ \\
\hline Kennedy e cols. (1998) (16) & 31 DM1 (insulina) & $\begin{array}{l}\text { Média glicêmica } \\
\text { Média pré-prandial } \\
\text { Média pós-prandial }\end{array}$ & $\begin{array}{c}\text { SMBG por duas semanas } \\
\text { antes }\end{array}$ & $\begin{array}{c}0,35(p<0,01) \\
0,104(N S) \\
0,36(p<0,01)\end{array}$ \\
\hline Delgado e cols. (2011) (15) & $\begin{array}{c}41 \text { DMG e DMPG (sem dados } \\
\text { do tratamento) }\end{array}$ & Glicemia em jejum (plasma) & $\begin{array}{c}\text { Glicemia mensurada junto com } \\
\text { a frutosamina }\end{array}$ & $r=0,63(p<0,001)$ \\
\hline Feitosa e cols. (2013) & $\begin{array}{l}47 \text { DM1, DM2 e DMG e } 71 \\
\text { frutosaminas }\end{array}$ & $\begin{array}{c}\text { Média glicêmica } \\
\text { DP } \\
\text { HBGl } \\
\text { LBGl } \\
\text { Glicemias pré e pós-prandiais }\end{array}$ & $\begin{array}{l}\text { SMBG por duas semanas } \\
\text { antes }\end{array}$ & $\begin{array}{c}\text { NS } \\
R=0,28(p=0,021) \\
R=0,26(p=0,030) \\
\text { NS } \\
\text { NS }\end{array}$ \\
\hline
\end{tabular}

N: número de pacientes; NS: não significativo; DM: diabetes melito; SMBG: self monitoring blood glucose = automonitoração da glicemia capilar; DMG: diabetes melito gestacional; DMGP: diabetes melito pré-gestacional; DM2: diabetes melito tipo 2; DMT1: diabetes melito tipo 1; DP: desvio-padrão; LGBI: low blood glucose index, HBGl: high blood glucose index. 
tras populacionais, tipo de tratamento, estabilidade do controle glicêmico, trimestre da gestação em que foi coletada a frutosamina e métodos de avaliação do perfil glicêmico e da frutosamina. Por exemplo, se a amostra populacional tivesse o controle glicêmico adequado e sem variabilidade, a frutosamina apresentaria correlação mais forte com a média glicêmica e mais fraca com os parâmetros de variabilidade e vice-versa.

O presente estudo traz como aspecto relevante a análise da correlação da frutosamina com parâmetros adicionais extraídos da automonitoração glicêmica para avaliação da variabilidade e risco de excursões hiper e hipoglicêmicas. Nossos resultados revelaram correlação direta entre a frutosamina e o desvio-padrão das glicemias e o HBGI nas gestantes diabéticas. Não encontramos outros estudos que tenham avaliado a frutosamina e esses parâmetros nas gestantes portadoras de diabetes. A frutosamina é o termo geral para descrever as proteínas glicadas totais (1), das quais 50 a $80 \%$ são albuminas $(1,9)$. A albumina glicada (AG) tem forte correlação com a frutosamina (32) e outros estudos demonstram correlacionar-se, em não gestantes, com parâmetros de hiperglicemia e variabilidade glicêmica. Matsumoto e cols. demonstraram que a AG reflete as flutuações da glicemia e as excursões glicêmicas pós-prandiais (33) e Chon e cols. (10) demonstraram que a frutosamina, em portadores de diabetes com bom controle glicêmico (HbAlc $<7 \%$ ), correlaciona-se com variabilidade (MAGE) e hiperglicemia pós-prandial (MPMG). Nossos achados estão concordantes com ambos os estudos. O fato de a AG aumentar conforme se elevam as concentrações de glicose (34) e a taxa de glicação da albumina glicada ser 10 vezes maior que a da hemoglobina (35) pode justificar que as concentrações séricas da AG, e consequentemente a frutosamina, também possam ser influenciadas pelos picos hiperglicêmicos temporários (36).

Nosso estudo também revelou que, em neonatos PIG com valores de frutosamina semelhantes aos neonatos AIG e GIG, houve maior LBGI e maior frequência de glicemias abaixo de $60 \mathrm{mg} / \mathrm{dL}$. Esses dados sugerem que a frutosamina, mesmo dentro do intervalo de referência da normalidade para gestação, em neonatos PIG, subestimam hipoglicemias. Provalvemente o período em hiperglicemia foi maior e pôde ser representado pela frutosamina, que se mostrou associada com o DP e o HBGI, entretanto, as hipoglicemias, com menor duração, ainda que frequentes, não puderam ser representadas pela frutosamina. O achado de feto PIG ou com peso limítrofe durante a gestação de portadora de diabetes com frutosamina normal poderia indicar a necessidade de monitoração mais frequente das glicemias capilares em gestantes a fim de caracterizar melhor o perfil glicêmico. Uma limitação do estudo foi o pequeno número de neonatos analisados, o que poderia reduzir a robustez das diferenças encontradas. Estudos com maior número de neonatos são necessários para confirmar os achados.

Os ensaios para frutosamina são rápidos e de baixo custo e podem ser feitos rotineiramente nos laboratórios, entretanto são subutilizados. Nas situações em que a HbAlc apresenta limitações (gravidez, doença renal crônica, hemoglobinopatias), a frutosamina pode ser utilizada quando a automonitoração e a HbAlc estão em conflito (37). Tem sido sugerido que a frutosamina deva ser determinada como rotina em pacientes com concentrações de HbAlc normais para confirmar se estão em adequado controle (8).

Nosso estudo demonstrou que, em gestantes diabéticas, a frutosamina elevada relaciona-se a picos hiperglicêmicos e variabilidade e, naquelas com fetos PIG, correlaciona-se com hipoglicemias ocultas. A frutosamina isolada não deve ser usada como parâmetro único para o controle glicêmico na gravidez, porém, quando elevada ou mesmo próxima ao limite superior, pode indicar maior necessidade de monitorar os picos pós-prandiais e, quando normal em RNs PIG, indicar maior atenção para hipoglicemias ocultas. Estudos adicionais são necessários para identificar biomarcadores alternativos e apropriados para avaliar a hiperglicemia pós-prandial e a variabilidade glicêmica em gestantes.

Declaração: os autores declaram não haver conflitos de interesse científico neste estudo.

\section{REFERÊNCIAS}

1. Armbruster DA. Fructosamine: structure, analysis, and clinical usefulness. Clin Chem. 1987;33(12):2153-63.

2. Koga M, Kasayama S. Clinical impact of glycated albumin as another glycemic control marker. Endocr J. 2010;57(9):751-62.

3. Dolhofer $\mathrm{R}$, Wieland $\mathrm{OH}$. Increased glycosylation of serum albumin in diabetes mellitus. Diabetes. 1980;29(6):417-22.

4. Baker JR, Metcalf PA, Johnson RN, Newman D, Rietz P. Use of protein-based standards in automated colorimetric determinations of fructosamine in serum. Clin Chem. 1985;31(9):1550-4.

5. Pandya HC, Livingstone S, Colgan M, Percy R, Frier B. Serum fructosamine as an index of glycaemia: comparison with glycated haemoglobin in diabetic and non-diabetic individuals. Pract Diab Int. 1987;4(3):126-8. 
6. Narbonne H, Renacco E, PradelV, Portugal H, Vialettes B. Can fructosamine be a surrogate for $\mathrm{HbA} 1 \mathrm{c}$ in evaluating the achievement of therapeutic goals in diabetes? Diabetes Metab. 2001;27(5):598-603.

7. Sociedade Brasileira de Diabetes S. Diretriz da Sociedade Brasileira de Diabetes 2009. 3.ed. Itapevi-SP: A Araújo Silva Farmacêutica; 2009.

8. Youssef D, El Abbassi A, Jordan RM, Peiris AN. Fructosamine: an underutilized tool in diabetes management: case report and literature review. Tenn Med. 2008;101(11):31-3.

9. Guthrow CE, Morris MA, Day JF, Thorpe SR, Baynes JW. Enhanced nonenzymatic glucosylation of human serum albumin in diabetes mellitus. Proc Natl Acad Sci U S A. 1979;76 (9):4258-61.

10. Chon S, LeeYJ, Fraterrigo G, Pozzilli P, Choi MC, Kwon MK, et al. Evaluation of glycemic variability in well-controlled type 2 diabetes mellitus. Diabetes Technol Ther. 2013;15(6):455-60.

11. Shafi T, Sozio SM, Plantinga LC, Jaar BG, Kim ET, Parekh RS, et al. Serum fructosamine and glycated albumin and risk of mortality and clinical outcomes in hemodialysis patients. Diabetes Care. 2013;36(6):1522-33

12. Roberts $A B, B a k e r ~ J R$. Relationship between fetal growth and maternal fructosamine in diabetic pregnancy. Obstet Gynecol. 1987;70:242-6.

13. Ismail NA, Olaide Raji $H$, Abd Wahab N, Mustafa N, Kamaruddin NA, Abdul Jamil M. Glycemic Control among Pregnant Diabetic Women on Insulin Who Fasted During Ramadan. Iran J Med Sci. 2011;36(4):254-9.

14. Koskinen P, Erkkola R, Viikari J, Mattila K, Irjala K. Blood glycated haemoglobin, serum fructosamine, serum glycated albumin and serum glycated total protein as measures of glycaemia in diabetes mellitus. Scand J Clin Lab Invest. 1992;52(8):863-9.

15. Delgado MR, Novik AV, Cardemil MF, Santander AD. [Plasma fructosamine to evaluate metabolic control among women with gestational diabetes]. Rev Med Chil. 2011;139(11):1444-50.

16. Kennedy DM, Johnson AB, Hill PG. A comparison of automated fructosamine and $\mathrm{HbA} 1 \mathrm{c}$ methods for monitoring diabetes in pregnancy. Ann Clin Biochem. 1998;35(Pt 2):283-9.

17. Parfitt VJ, Clark JD, Turner GM, Hartog M. Use of fructosamine and glycated haemoglobin to verify self blood glucose monitoring data in diabetic pregnancy. Diabet Med. 1993;10(2):162-6.

18. International Association of Diabetes and Pregnancy Study Groups Consensus Panel, Metzger BE, Gabbe SG, Persson B, Buchanan TA, Catalano PA, Damm P, et al. International Association of Diabetes and Pregnancy Study Groups Recommendations on the Diagnosis and Classification of Hyperglycemia in Pregnancy. Diabetes Care. 2010;33(3):676-82.

19. Lubchenco LO, Hansman C, Dressler M, Boyd E. Intrauterine growth as estimated from liveborn birth-weight data at 24 to 42 weeks of gestation. Pediatrics. 1963;32:793-800.

20. Crowther CA, Hiller JE, Moss JR, McPhee AJ, Jeffries WS, Robinson JS; Australian Carbohydrate Intolerance Study in Pregnant Women (ACHOIS) Trial Group. Effect of treatment of gestational diabetes mellitus on pregnancy outcomes. N Engl J Med. 2005;352(24):2477-86.

21. Rowan JA, Hague WM, Gao W, Battin MR, Moore MP; MiG Trial Investigators. Metformin versus insulin for the treatment of gestational diabetes. N Engl J Med. 2008;358(19):2003-15.

22. Kovatchev BP, Otto E, Cox D, Gonder-Frederick L, Clarke W. Evaluation of a new measure of blood glucose variability in diabetes. Diabetes Care. 2006;29(11):2433-8.
23. Cox DJ, Kovatchev BP, Julian DM, Gonder-Frederick LA, Polonsky $\mathrm{WH}$, Schlundt DG, et al. Frequency of severe hypoglycemia in insulin-dependent diabetes mellitus can be predicted from self-monitoring blood glucose data. J Clin Endocrinol Metab. 1994;79(6):1659-62.

24. Kovatchev BP, Straume M, Farhy LS, Cox DJ. Dynamic network model of glucose counterregulation in subjects with insulin-requiring diabetes. Methods Enzymol. 2000;321:396-410.

25. Kovatchev BP, Clarke WL, Breton M, Brayman K, McCall A. Quantifying temporal glucose variability in diabetes via continuous glucose monitoring: mathematical methods and clinical application. Diabetes Technol Ther. 2005;7(6):849-62.

26. Kovatchev BP, Cox DJ, Kumar A, Gonder-Frederick L, Clarke WL. Algorithmic evaluation of metabolic control and risk of severe hypoglycemia in type 1 and type 2 diabetes using self-monitoring blood glucose data. Diabetes Technol Ther. 2003;5(5):817-28.

27. Tertti K, Ekblad U, Koskinen P, Vahlberg T, RonnemaaT. Metformin vs. insulin in gestational diabetes. A randomized study characterizing metformin patients needing additional insulin. Diabetes Obes Metab. 2013;15(3):246-51.

28. Nor Azlin MI, Adam R, Sufian SS, Wahab NA, Mustafa N, Kamaruddin NA, et al. Safety and tolerability of once or twice daily neutral protamine hagedorn insulin in fasting pregnant women with diabetes during Ramadan. J Obstet Gynaecol Res. 2011;37(2):132-7.

29. Thai AC, Lui KF, Low EG, Ng WY, Kek LP. Serial measurement of serum fructosamine and glycosylated haemoglobin as indices of glycaemic control in diabetic pregnancy. Ann Acad Med Singapore. 1991;20(6):732-5.

30. Pasi KJ, Toop MJ, Cockrill BL, Hale PJ, Barnett AH, Wright AD. Serum fructosamine in diabetic pregnancy. Diabete Metab. 1989;15(4):151-5.

31. Watson WJ, Herbert WN, Prior TW, Chapman JF. Glycosylated hemoglobin and fructosamine. Indicators of glycemic control in pregnancies complicated by diabetes mellitus. J Reprod Med. 1991;36(10):731-4.

32. Beck R, Steffes M, Xing D, Ruedy K, Mauras N, Wilson DM, et al. The interrelationships of glycemic control measures: HbA1c, glycated albumin, fructosamine, 1,5-anhydroglucitrol, and continuous glucose monitoring. Pediatr Diabetes. 2011;12(8):690-5.

33. Matsumoto H, Murase-Mishiba Y, Yamamoto N, Sugitatsu-Nakatsukasa S, Shibasaki S, Sano H, et al. Glycated albumin to glycated hemoglobin ratio is a sensitive indicator of blood glucose variability in patients with fulminant type 1 diabetes. Intern Med. 2012;51(11):1315-21.

34. Kisugi R, Kouzuma T, Yamamoto T, Akizuki S, Miyamoto H, Someya $Y$, et al. Structural and glycation site changes of albumin in diabetic patient with very high glycated albumin. Clin Chim Acta. 2007;382(1-2):59-64.

35. Day JF, Thornburg RW, Thorpe SR, Baynes JW. Nonenzymatic glucosylation of rat albumin. Studies in vitro and in vivo. J Biol Chem. 1979;254(19):9394-400.

36. Ogawa A, Hayashi A, Kishihara E, Yoshino S, Takeuchi A, Shichiri $\mathrm{M}$. New indices for predicting glycaemic variability. PloS One. 2012;7(9):e46517.

37. Hirsch IB, Amiel SA, Blumer IR, Bode BW, Edelman SV, Seley JJ, et al. Using multiple measures of glycemia to support individualized diabetes management: recommendations for clinicians, patients, and payers. Diabetes TechnolTher. 2012;14(11):973-83; quiz 83. 